Saclay-T94/041

\title{
GAUGE STRUCTURE AND SEMI-CLASSICAL ASPECTS OF HARD THERMAL LOOPS.
}

\author{
Jean-Paul BLAIZOT and Edmond IANCU \\ Service de Physique Théoriquel, CE-Saclay \\ 91191 Gif-sur-Yvette, France
}

April 1994

\begin{abstract}
Hard thermal loops play a central role in the theory of long wavelength excitations of a quark-gluon plasma. We show in this paper how our recent derivation of their generating functional from the Dyson-Schwinger equations sheds light on their semi-classical nature and their remarkable gauge structure. In particular, we show that our kinetic equations can be written in terms of auxiliary gauge fields with zero curvature. Remarkably, the latter property determines entirely the dynamics in the kinematical regime of hard thermal loops. This explains in particular why the generating functional could have been obtained by Taylor and Wong from gauge invariance considerations. It also clarifies the role of Chern-Simons theory in this context.
\end{abstract}

\footnotetext{
${ }^{*}$ CNRS

†Laboratoire de la Direction des Sciences de la Matière du Commissariat à l'Energie Atomique
} 


\section{Introduction}

"Hard thermal loops" (HTL) are the dominant contributions at high temperature to $n$ point amplitudes with soft external momenta $(P \sim g T$, with $T$ the temperature and $g$ the gauge coupling, assumed to be small). Their role in perturbative calculations, and their remarkable properties, where first established via a systematic diagrammatic analysis [1, 2, 3]. One of their most noticeable property is their gauge symmetry; specifically, hard thermal loops obey Ward identities similar to those of the tree amplitudes, suggesting that their generating functional should be a gauge invariant functional of the soft fields. The gauge invariance has been used by Taylor and Wong to construct a generating functional for the HTL's in closed form[1. Later, this effective action has been rewritten in a manifestly gauge invariant form by Braaten and Pisarski[5] and also by Frenkel and Taylor [6]. It was also realized that the generating functional of HTL's could be understood as the eikonal of a Chern-Simons gauge theory, raising the hope that the use of the known properties of the latter theory could lead to a better understanding of gauge theories at finite temperature[7].

In a series of recent papers, we have shown that the hard thermal loops are intimately related to the long wavelength, collective, excitations of the hot gauge plasmas, much alike the non trivial dielectric properties of the electromagnetic plamas are related to the collective oscillations of their charge constituents. To establish this correspondence, we have developed a theory of the collective excitations, which is gauge covariant and takes consistently into account all the leading terms in an expansion of the equations of motion in power of the coupling strength [8, 9, [10].

A central quantity in our approach is the "induced current" which represents the response of the plasma particles to applied background gauge fields. This is obtained, after solving nonlinear equations which may be given the form of kinetic equations, as a non local functional of the gauge fields. The nonlinearity of these equations is entirely due to gauge rotations of the current (a vector in color space) induced by covariant derivatives. The non locality of the induced current arises from the need, in calculating the response functions, to integrate the history of the hard particles along their straight line trajectories. These simple features, whose physical origin is tranparent, explain most of the properties of the HTL's, properties which remained somewhat surprising in the diagrammatic approach.

In this paper we wish to pursue our analysis, and make closer contact with the formal works mentioned above. In particular we shall show that the gauge structure of the HTL's follows from a simple property of our kinetic equations, which is ultimately 
related to a property of the Lorentz equation describing the motion of a classical particle in an electromagnetic field, or its generalisation to the non abelian case. More precisely, we shall show that, in the same way that the Lorentz equation can be rewritten in terms of the gauge dependent canonical momentum, our kinetic equations can be also written in terms of auxiliary gauge potentials with zero field strength. The latter property follows from the fact that, within the present approximation scheme, the plasma particles do not change direction in the course of their interaction with the gauge field. Quite remarkably, in this kinematical regime, the condition of zero field strength turns out to be sufficient to determine the dynamics. As we shall see, this provides an explanation for the fact that it has been possible to construct the generating functional for the hard thermal loops on the basis of gauge invariance only, with no dynamical input beyond the form of the gluon self energy [4, [5].

The plan of the paper is as follows. In the next section, we present a short summary of our approach, and recall the form of our basic kinetic equations. In section 3 we discuss some of their semi-classical features, and show that they can be given a simple gauge structure. Then in section 4 we show explicitely how the gauge invariance condition used by Taylor and Wong to derive the generating functional of HTL follows from our kinetic equations. We also clarify the role of Chern-Simons theory whose relevance in the present context has been much advocated recently. Finally, we verify that the expression for the energy density obtained by Nair using Chern-Simons theory coincides with the one that we have obtained by integrating the mean field equations. The last section summarizes our conclusions.

\section{Semiclassical theory of collective excitations}

Our semi-classical theory of the collective excitations is based on a perturbative analysis of the QCD Dyson-Schwinger equations. It is obtained by consistently preserving the leading terms in an expansion in powers of the gauge coupling $g$. In doing so, one encounters three types of approximations, which, in most many-body systems, are independent approximations, but here are controlled by the same small parameter, i.e. $g$. These are the weak coupling approximation $(g \ll 1)$, the long wavelength approximation $(\lambda \sim 1 / g T \gg 1 / T)$, and the small amplitude approximation (the gauge field strength tensor is limited by $F \lesssim g T^{2}$, or, equivalently, the gauge potentials satisfy $A \lesssim T$ ).

The result of our analysis is a set of coupled equations. The first equation, the generalization of the Maxwell equation in a polarizable medium, relates the gauge mean field 
$A_{\mu}^{a}$ to the induced current $j_{\mu}^{a}$ (see (a) below). The other equations allow the calculation of the induced current in terms of the average gauge fields. They can be given the form of simple kinetic equations, analogous to Vlasov's equation of ordinary plasma physics (see (c) below). In our approach, the hard thermal loops appear as functional derivatives of the induced current with respect to the gauge fields [8, 9, 10].

(a) Mean field equation

The equation of motion for $A_{\mu}^{a}$ takes the form

$$
\left[D^{\nu}, F_{\nu \mu}(x)\right]^{a}=j_{\mu}^{a}(x),
$$

where $D_{\mu}=\partial_{\mu}+i g A_{\mu}(x),\left(A_{\mu} \equiv A_{\mu}^{a} t^{a}\right), F_{\mu \nu}=\left[D_{\mu}, D_{\nu}\right] /(i g)$ is the field strength tensor, and $j_{\mu}(x)$ is the induced current, which transforms as a color vector in the adjoint representation. (The generators of the gauge group in different representations are taken to be Hermitian and traceless. They are denoted by $t^{a}$ and $T^{a}$, respectively, for the fundamental and the adjoint representation, and are normalized so that $\operatorname{Tr}\left(t^{a} t^{b}\right)=(1 / 2) \delta^{a b}$ and $\operatorname{Tr}\left(T^{a} T^{b}\right)=N \delta^{a b}$.)

(b) Structure of the induced current

The induced current expresses the response of the plasma particles to the long wavelength color fields $A_{\mu}^{a}$. It is convenient to separate the contributions of the fermions from those of the transverse gluons, by writing $j_{\mu}^{a}=j_{\mathrm{f} \mu}^{a}+j_{\mathrm{b} \mu}^{a}$. For the fermions (with $N_{f}$ flavors) we have

$$
j_{\mathrm{f} \mu}^{a}(x)=g N_{f} \int \frac{d^{3} k}{(2 \pi)^{3}} v_{\mu}\left(\delta n_{+}^{a}(\mathbf{k}, x)-\delta n_{-}^{a}(\mathbf{k}, x)\right),
$$

where $v^{\mu} \equiv(1, \mathbf{v})$ and $\mathbf{v} \equiv \mathbf{k} / k$ is the velocity of the hard particle $(k \equiv|\mathbf{k}|$, and $|\mathbf{v}|=1)$. The quantities $\delta n_{ \pm}^{a}(\mathbf{k}, x)$ are the components of a color vector in the adjoint representation. They may be combined into a density matrix, $\delta n_{ \pm} \equiv \delta n_{ \pm}^{a} t^{a}$, ultimately related to the fermion propagator in the presence of the gauge fields. There is no color singlet component in $\delta n_{ \pm}$because we are considering only excitations which carry gluon quantum numbers.

Similarly, the current carried by the transverse gluons can be written in terms of a density matrix $\delta N(\mathbf{k}, x) \equiv \delta N^{a}(\mathbf{k}, x) T^{a}$ :

$$
j_{\mathrm{b} \mu}^{a}(x)=2 g \int \frac{d^{3} k}{(2 \pi)^{3}} v_{\mu} \operatorname{Tr} T^{a} \delta N(\mathbf{k}, x)=2 g N \int \frac{d^{3} k}{(2 \pi)^{3}} v_{\mu} \delta N^{a}(\mathbf{k}, x) .
$$

(c) Kinetic equations for $\delta n_{ \pm}$and $\delta N$ 
The color density matrices $\delta n_{ \pm}$and $\delta N$ are determined by the following kinetic equations 8 , 10

$$
\begin{aligned}
& {\left[v \cdot D_{x}, \delta n_{ \pm}(\mathbf{k}, x)\right]=\mp g \mathbf{v} \cdot \mathbf{E}(x) \frac{d n_{k}}{d k},} \\
& {\left[v \cdot D_{x}, \delta N(\mathbf{k}, x)\right]=-g \mathbf{v} \cdot \mathbf{E}(x) \frac{d N_{k}}{d k}}
\end{aligned}
$$

where $E_{a}^{i} \equiv F_{a}^{i 0}$ is the chromoelectric field. Furthermore, $N_{k} \equiv 1 /(\exp (\beta k)-1)$ and $n_{k} \equiv 1 /(\exp (\beta k)+1)$ are, respectively, the equilibrium boson and fermion occupation factors. Note that, in deriving these equations, we have used the isotropy (in $\mathbf{k}$ space) of the equilibrium distribution functions. For more general, anisotropic, equilibrium distributions $n(\mathbf{k})$ and $N(\mathbf{k})$, we should replace

$$
\mathbf{v} \cdot \mathbf{E}(x) \frac{d n_{k}}{d k} \longrightarrow F^{i \nu}(x) v_{\nu} \frac{\partial n}{\partial k^{i}}
$$

in the r.h.s. of eq. (2.4), and similarly for the second equation.

In the abelian case, eq.(2.4) reduces to the well known linearized Vlasov equation describing the evolution of single particle distribution functions. It is perhaps useful here to emphasize that, although the underlying dynamics is that of the Lorentz equation describing the motion of a classical charged particle in an electromagnetic field (see next section), however $\delta n(\mathbf{k}, x)$ is a Wigner function whose only slow variations in $x$ have a classical character (in particular, the plasma particles have typical wavelength comparable to their mean separation and remain therefore quantum particles). In the non abelian case, the distribution functions are matrices in color space: the color degree of freedom remains essentially quantum. (In this respect we differ from Heinz who has given a derivation of equations similar to ours starting from classical kinetic theory, and treating color also as a classical degree of freedom 11.)

For the subsequent analysis of these equations, it is convenient to express $\delta n_{ \pm}$and $\delta N$ in terms of new functions, $W^{\mu}(x ; v) \equiv W_{a}^{\mu}(x ; v) t^{a}$, defined as the solutions to

$$
\left[v \cdot D_{x}, W^{\mu}(x ; v)\right]=F^{\mu \nu}(x) v_{\nu}
$$

The quantities $W_{a}^{\mu}(x ; v)$ are the components of a color vector in the adjoint representation, and satisfy

$$
v_{\mu} W_{a}^{\mu}(x ; v)=0
$$

so that $W_{a}^{0}=v^{i} W_{a}^{i}$. From the equations above, it is easily seen that

$$
\delta n_{ \pm}^{a}(\mathbf{k}, x)=\mp g W_{a}^{0}(x ; v) \frac{d n}{d k}, \quad \delta N^{a}(\mathbf{k}, x)=-g W_{a}^{0}(x ; v) \frac{d N}{d k} .
$$


Note that for an anisotropic initial distribution, the fluctuations $\delta n_{ \pm}^{a}$ and $\delta N^{a}$ depend on all the three independent components of $W_{a}^{\mu}(x ; v)$, that is, e.g.,

$$
\delta n_{ \pm}^{a}(\mathbf{k}, x)=\mp g W_{a}^{i}(x ; v) \frac{\partial n}{\partial k^{i}}
$$

and similarly for $\delta N^{a}$.

(d) Solving the kinetic equations

The equation (2.6) for $W_{a}^{\mu}$ is easily solved once boundary conditions are given. We assume here retarded conditions, such that the fields $A_{\mu}$ vanish as $x_{0} \rightarrow-\infty$. The corresponding solution of eq. (2.6) reads

$$
W^{\mu}(x ; v)=\int_{0}^{\infty} d \tau U(x, x-v \tau) F^{\mu \nu}(x-v \tau) v_{\nu} U(x-v \tau, x),
$$

where $U(x, y)$ is the parallel transporter along the straight line $\gamma$ joining $x$ and $y$,

$$
U(x, y)=P \exp \left\{-i g \int_{\gamma} d z^{\mu} A_{\mu}(z)\right\}
$$

with $P$ denoting the path-ordering operator. Thus,

$$
U(x, x-v \tau)=P \exp \left\{-i g \int_{0}^{\tau} d s v \cdot A(x-v(\tau-s))\right\} .
$$

In order to verify that (2.9) is the correct solution to eq. (2.6) we may use the following formula for the line-derivative of the parallel transporter

$$
\left.\left(v \cdot \partial_{x}\right) U(x, y)\right|_{y=x-v \tau}=-i g v \cdot A(x) U(x, x-v \tau) .
$$

We shall use later the quantity

$$
V(x ; v) \equiv \lim _{\tau \rightarrow \infty} U(x, x-v \tau)
$$

which is well defined since $A_{\mu}(x-v \tau) \rightarrow 0$ as $\tau \rightarrow \infty$. We have

$$
(v \cdot \partial) V(x ; v)=-i g v \cdot A(x) V(x ; v)
$$

which leads to the following representation for $v \cdot A(x)$ :

$$
v \cdot A(x)=\frac{i}{g}((v \cdot \partial) V) V^{-1} .
$$

The matrix $V(x ; v)$ generates the gauge transformation from the gauge $v \cdot A=0$ to the actual gauge.

(e) Induced current in closed form 
Once the solution of the kinetic equation is known, one can easily calculate the induced current in closed form. By inserting eqs. (2.8) in the expressions (2.2) and (2.3), we obtain

$$
j_{a}^{\mu}(x)=3 \omega_{p}^{2} \int \frac{d \Omega}{4 \pi} v^{\mu} W_{a}^{0}(x ; v) .
$$

after the integration over $k=|\mathbf{k}|$ has been performed. Here $\omega_{p}$ is the plasma frequency, $\omega_{p}^{2} \equiv\left(2 N+N_{\mathrm{f}}\right) g^{2} T^{2} / 18$. The integral $\int d \Omega$ runs over all the directions of the unit vector v. According to eqs. (2.9) and (2.16), the retarded current reads

$$
j^{\mu}(x)=3 \omega_{p}^{2} \int \frac{d \Omega}{4 \pi} v^{\mu} \int_{0}^{\infty} d \tau U(x, x-v \tau) \mathbf{v} \cdot \mathbf{E}(x-v \tau) U(x-v \tau, x) .
$$

This expression can be viewed as a generating functional of the hard thermal loops with retarded boundary conditions. It was first presented in Ref. [9], and has been rederived since using other methods 13 (see also below). The current (2.16) is covariantly conserved,

$$
\left[D_{\mu}, j^{\mu}(x)\right]=0
$$

as necessary for the consistency of the mean field equation (2.1). The "abelian-like" Ward identities relating the HTL's, which were first identified in the diagrammatic approach of Refs. [2, 3], are a direct consequence of eq. (2.18).

\section{Gauge structure}

We turn now to a physical interpretation of some of the quantities that we have introduced, and to an analysis of the simple gauge structure which emerges from our equations.

First, it is useful to recall various forms of the equations of motion for a classical particle of mass $m$ and charge $e$, moving in an electromagnetic background field. From the Lagrange function 12

$$
L=-m \sqrt{1-\mathbf{v}^{2}}-e v \cdot A,
$$

one easily obtains $\left(v^{\mu} \equiv(1, \mathbf{v})\right)$

$$
\frac{d \mathbf{p}}{d t}=-e v^{\mu} \nabla A_{\mu} .
$$

The canonical momentum

$$
\mathbf{p}=\frac{\partial L}{\partial \mathbf{v}}=\frac{m \mathbf{v}}{\sqrt{1-\mathbf{v}^{2}}}+e \mathbf{A} \equiv \mathbf{k}+e \mathbf{A}
$$


is the sum of the kinetic momentum $\mathbf{k}$, related to the velocity of the particle, and $e$ times the gauge field $\mathbf{A}$. The energy of the particle may be given a similar form,

$$
p^{0}=\mathbf{p} \cdot \mathbf{v}-L=\frac{m}{\sqrt{1-\mathbf{v}^{2}}}+e A^{0} \equiv k^{0}+e A^{0}
$$

It obeys the equation

$$
\frac{d p^{0}}{d t}=e v^{\mu} \partial_{0} A_{\mu}
$$

so that, for the 4-momentum $p^{\mu}$, we have:

$$
\frac{d p^{\mu}}{d t}=e v^{\nu} \partial^{\mu} A_{\nu}
$$

This equation is not manifestly gauge covariant. However, the equation for the kinetic 4-momentum $k^{\mu}=p^{\mu}-e A^{\mu}$,

$$
\frac{d k^{\mu}}{d t}=e F^{\mu \nu}(x) v_{\nu}
$$

is explicitly independent of the choice of the gauge. We shall see that, to the two ways of writing the Lorentz equation, namely eqs. (3.6) and (3.7), correspond two ways of writing our basic kinetic equation (2.6) for $W^{\mu}$.

Consider first a QED plasma, where eq. (2.6) reduces to

$$
\left(v \cdot \partial_{x}\right) W^{\mu}(x ; v)=F^{\mu \nu}(x) v_{\nu}
$$

This is very much similar to eq. (3.7). To make the analogy closer, we remark that the line derivative in the l.h.s. of eq. (3.8), i.e. $v \cdot \partial_{x}=\partial_{t}+\mathbf{v} \cdot \boldsymbol{\nabla}$, may be interpreted as the total time derivative along the trajectory $\mathbf{x}(t)=\mathbf{x}_{0}+\mathbf{v} t$ of a fictitious particle. That is, eq. (3.8) may be rewritten as

$$
\frac{d}{d t} W^{\mu}(t, \mathbf{x}(t) ; v)=F^{\mu \nu}(t, \mathbf{x}(t)) v_{\nu}
$$

showing that $e d W^{\mu}(x ; v)$ is the kinetic 4-momentum acquired during the time $d t$ by a charged particle due to its interaction with the electromagnetic field. Note, however, that in calculating $W^{\mu}$ according to eq. (2.9) we have assumed the velocity $\mathbf{v}$ to be constant, while in the Lorentz equation (3.7), $k^{\mu}$ and $\mathbf{v}$ are related quantities $\left(k^{\mu}=k^{0} v^{\mu}\right)$. This is in line with our assumption that the typical momenta of the thermal particles are "hard", $k \sim T$, while those of the background fields are "soft", $P \sim g T \ll k$. Thus the hard particles follow straight line trajectories at the speed of the light, and these trajectories are not altered, to leading order, by the interaction with the background gauge field. The 
condition (2.7) reflects then simply the fact that the energy transferred by the field, $e W^{0}$, coincides with the mechanical work done by the Lorentz force, $e \mathbf{v} \cdot \Delta \mathbf{k}=e v^{i} W^{i}$. With this interpretation, the formulae (2.8) for $\delta n_{ \pm}$become transparent:

$$
\begin{aligned}
\delta n_{ \pm}(\mathbf{k}, \mathbf{x}, t) & =\mp e W^{0} \frac{d n}{d k}=\mp e W^{i} \frac{\partial n}{\partial k^{i}}=-\Delta k_{ \pm}^{i} \frac{\partial n}{\partial k^{i}} \\
& \approx n\left(\mathbf{k}-\Delta \mathbf{k}_{ \pm}(\mathbf{x}, t)\right)-n(\mathbf{k}),
\end{aligned}
$$

that is, the particles found at time $t$ at the point $\mathbf{x}$ with momentum $\mathbf{k}$, are those which, in the initial distribution, have momentum $\mathbf{k}-\Delta \mathbf{k}(\mathbf{x}, t)$, where $\Delta \mathbf{k}(\mathbf{x}, t)$ is the momentum acquired by the particles whose trajectories go through $\mathbf{x}$ at time $t$.

In the non-abelian case, the fluctuations $\delta n_{ \pm}$and $\delta N$ are matrices in color space, that is, $W^{\mu}=W_{a}^{\mu} t^{a}$. The color vector of components $W_{a}^{\mu}$ precesses in the background gauge field. This precession is induced by the covariant derivative in eq. (2.6). Viewing this precession as an additional source of time-dependence for the color vector $W_{a}^{\mu}$, one can write

$$
\frac{d}{d t} W_{a}^{\mu}(t, \mathbf{x}(t) ; v)=\left[\left(\partial_{t}+\mathbf{v} \cdot \boldsymbol{\nabla}\right) \delta_{a c}-g f_{a b c}\left(v \cdot A_{b}\right)\right] W_{c}^{\mu},
$$

so that eq. (2.6) may be given a form similar to eq. (3.9).

We shall see now that it is possible to rewrite our kinetic equation in a form similar to eq. (3.6) for the canonical momentum. This follows by noticing that

$$
F^{\mu \nu} v_{\nu}=\partial^{\mu}(v \cdot A)-\left[v \cdot D, A^{\mu}\right] .
$$

Thus, if one sets

$$
a^{\mu}(x ; v) \equiv A^{\mu}(x)+W^{\mu}(x ; v),
$$

one gets

$$
\left[v \cdot D, a^{\mu}\right]=\partial^{\mu}(v \cdot A),
$$

showing that $a^{\mu}$ is a functional of $v \cdot A$ only. Explicitly,

$$
a^{\mu}(x ; v)=\int_{0}^{\infty} d \tau U(x, x-v \tau) \partial^{\mu}(v \cdot A(x-v \tau)) U(x-v \tau, x),
$$

where the retarded boundary conditions have been taken into account. In the abelian case, eq. (3.14) can be rewritten in a form analogous to eq. (3.6) for $p^{\mu}$ :

$$
\frac{d}{d t} a^{\mu}(t, \mathbf{x}(\mathbf{t}) ; v)=\left(\partial_{t}+\mathbf{v} \cdot \nabla\right) a^{\mu}=\partial^{\mu}(v \cdot A),
$$


showing that $g a^{\mu}$ may be indeed understood as the change in the canonical momentum $p^{\mu} \equiv k^{\mu}+g A^{\mu}$ of a fictitious particle following the trajectory $\mathbf{x}(t)=\mathbf{x}_{0}+\mathbf{v} t$.

The two writings of our kinetic equations, namely eq. (2.6) and eq. (3.14), have their respective advantages. Eq. (2.6) is manifestly gauge covariant, while eq. (3.14) is not. On the other hand, the new fields $a^{\mu}$ transform as gauge potentials, which has interesting consequences, as we shall see now. Under a gauge transformation induced by the operator $h(x)=\exp \left(-i g \theta^{a}(x) t^{a}\right), W^{\mu}=W_{a}^{\mu} t^{a}$ transforms as $W^{\mu}(x) \rightarrow h(x) W^{\mu}(x) h^{-1}(x)$, while $A_{\mu}=A_{\mu}^{a} t^{a} \rightarrow h A_{\mu} h^{-1}-(i / g) h \partial_{\mu} h^{-1}$. Thus $a^{\mu}(x ; v)$ transforms indeed as a gauge potential. Let $\mathcal{D}_{\mu} \equiv \partial_{\mu}+i g a_{\mu}$ be the covariant derivative constructed from $a_{\mu}$, and $f_{\mu \nu}(x ; v) \equiv\left[\mathcal{D}_{\mu}, \mathcal{D}_{\nu}\right] /(i g)$ the corresponding field strength tensor. Then it may be shown that

$$
f_{\mu \nu}=\partial_{\mu} a_{\nu}-\partial_{\nu} a_{\mu}+i g\left[a_{\mu}, a_{\nu}\right]=0
$$

To verify (3.17), one could directly derive the equation satisfied by $f_{\mu \nu}$, starting with eq. (3.14). This is the way followed in Ref. [17, where eq. (3.17) was first obtained (see eq. (3.24) in Ref. [17]). An alternative derivation is to solve eq. (3.14) for $a^{\mu}$, with $v \cdot A$ given by eq. (2.15). It is then easily seen that that

$$
a^{\mu}(x ; v)=\frac{i}{g}\left(\partial^{\mu} V\right) V^{-1}
$$

from which eq. (3.17) follows.

In summary, our basic dynamical equation can be written either in terms of $W^{\mu}$ (see eq. (2.6)), or in terms of $a^{\mu}$ (as in eq. (3.14)). In the abelian case, this freedom corresponds to the choice of writing the Lorentz equation in terms of the kinetic or of the canonical momentum, respectively. The fact that the field $a^{\mu}$ turns out to be a pure gauge (see eqs. (3.17) and (3.18) ) results ultimately from the assumption that the trajectories of the plasma particles are not deviated by the interaction with the gauge fields. In particular, $a^{\mu}$ can be set equal to zero by an appropriate choice of the gauge, specifically, by choosing the gauge $v \cdot A=0$ (see eq. (3.15)). Then, $W^{\mu}=-A^{\mu}$, as can be seen directly on eq. (2.6). Note, however, that for practical purposes, such a gauge is of no use, since the calculation of the induced current - the quantity one is primarily interested in involves an integration over all directions of $\mathbf{v}$ (see eq. (2.16)).

Quite remarkably, the gauge structure that we have exhibited completely determines the underlying dynamics. This explains why most of the dynamical information could have been reconstructed from arguments based on gauge invariance only 4 . To see this, we note that the dynamical equation (3.14) for $a^{\mu}=A^{\mu}+W^{\mu}$ follows from eq. (3.17), once 
the condition (2.7) is taken into account. Indeed, by multiplying eq. (3.17) by $v^{\nu}$, we obtain

$$
(v \cdot \partial) a_{\mu}-v^{\nu}\left(\partial_{\mu} a_{\nu}\right)+i g\left[v \cdot a, a_{\mu}\right]=0
$$

Using the fact that $v^{\mu}$ is a constant vector, together with the property $v \cdot a=v \cdot A$ (which follows from eqs. (2.7) and (3.13)), we can write this equation in the form (3.14). To the extent that one is interested only in the calculation of the induced current - which, according to eq. (2.16), depends on $W^{0}=a^{0}-A^{0}$ - only the component $\mu=0$ of eq. (3.14) is needed. Explicitly,

$$
(v \cdot \partial) a_{0}-\partial_{0}(v \cdot A)+i g\left[v \cdot A, a_{0}\right]=0 .
$$

This is, within minor notational changes, the equation which has been obtained by Taylor and Wong as the condition of gauge invariance of the effective action [4. This is discussed in detail in the next section.

\section{Relation to previous works}

As we have seen, in the computation of the induced current, the important gauge variables are $A^{0}$ and $v \cdot A=A^{0}-\mathbf{v} \cdot \mathbf{A}$. (Recall that $a^{0}$ is a functional of $v \cdot A$ only, as implied by eq. (3.20).) Alternatively, and in order to facilitate the comparaison with previous works, it is convenient to use the variables $A_{+} \equiv v \cdot A$ and $A_{-} \equiv v^{\prime} \cdot A$, where $v^{\mu}=(1, \mathbf{v})$ and $v^{\prime \mu}=(1,-\mathbf{v})$. We also set $\partial_{+} \equiv v \cdot \partial$ and $\partial_{-} \equiv v^{\prime} \cdot \partial$. In these notations, eq. (3.20) reads

$$
\partial_{+} a_{0}-\partial_{0} A_{+}+i g\left[A_{+}, a_{0}\right]=0 .
$$

We show now that this is precisely the condition for the gauge invariance of the generating functional of the HTL's, denoted by $S\left[A_{\mu}\right]$. To do this, let us recall that the induced current is, formally, the first derivative of the effective action $S$,

$$
\frac{\delta S}{\delta A_{\mu}^{a}(x)}=j_{a}^{\mu}(x)=3 \omega_{p}^{2} \int \frac{d \Omega}{4 \pi} v^{\mu} W_{a}^{0}(x ; v) .
$$

Here, "formally" means that in writing eq. (4.2) we are renouncing to the retarded conditions for $A_{\mu}$ and $j^{\mu}$. Indeed, eq. (4.2) implies

$$
\frac{\delta j_{a}^{\mu}(x)}{\delta A_{\nu}^{b}(y)}=\frac{\delta j_{b}^{\nu}(y)}{\delta A_{\mu}^{a}(x)}
$$


which can only hold on the space of fields in which the operator $v \cdot D$ is invertible 4 , 10. In this functional space, which we denote by $\mathcal{R}$, we can integrate (4.2) to get $S[A]$. Setting $W^{0}=a^{0}-A^{0}$, and separating the contributions of $a^{0}$ and $A^{0}$, we write eq. (4.2) as

$$
\frac{\delta S}{\delta A_{\mu}^{a}(x)}=3 \omega_{p}^{2}\left\{-g^{\mu 0} A_{0}^{a}+\int \frac{d \Omega}{4 \pi} v^{\mu} a_{a}^{0}\left[A_{+}\right]\right\},
$$

where we have indicated explicitly that $a^{0}$ depends only on $A_{+} \equiv v \cdot A$. The effective action is therefore of the form:

$$
S=3 \omega_{p}^{2} \int d^{4} x\left\{-\frac{1}{2} A_{a}^{0} A_{a}^{0}+\int \frac{d \Omega}{4 \pi} \mathcal{W}\left[A_{+}\right]\right\},
$$

where, by construction, the functional $\mathcal{W}$ satisfies

$$
\frac{\delta \mathcal{W}}{\delta A_{+}^{a}(x)}=a_{a}^{0}(x ; v)
$$

Consider now an infinitesimal gauge transformation

$$
A_{\mu} \rightarrow A_{\mu}+\delta A_{\mu}, \quad \delta A_{\mu}=\left[D_{\mu}, \theta\right]
$$

Under such a transformation, $S \rightarrow S+\delta S$, with

$$
\delta S=3 \omega_{p}^{2} \int d^{4} x \int \frac{d \Omega}{4 \pi} \theta^{a}\left\{\partial_{0} A_{+}^{a}-\partial_{+} a_{0}^{a}+g f^{a b c} A_{+}^{b} a_{0}^{c}\right\} .
$$

Since $a_{0}^{a}$ satisfies eq. (4.1), it follows that $\delta S=0$.

The form of the effective action given by eq. (4.5) was first inferred by Taylor and Wong from an analysis of the HTL's 1 . By assuming $S$ to be gauge invariant, they obtained eq. (4.1) as an equation for $\delta W / \delta A_{+}$. And, in fact, our arguments above have been intentionally developed in a way which makes the relation to the analysis of Taylor and Wong obvious. But, of course, we could directly infer the gauge invariance of $S$ from the conservation of the induced current, through a standard, Noether-type, argument. That is, by using eqs. (4.2) and (4.7), we can write

$$
\delta S=\int d^{4} x j_{a}^{\mu} \delta A_{\mu}=-\int d^{4} x \theta^{a}\left[D_{\mu}, j^{\mu}\right]_{a},
$$

where the second equality follows after an integration by parts (the surface term has been assumed to vanish). Because of the conservation law for $j^{\mu}$, eq. (2.18), the last integral vanishes, and so does $\delta S$. The relation between the condition for the gauge invariance of the effective action and the dynamical equation for $j^{\mu}$ has also been pointed out in Ref. 18. 
The arguments above suggest a different way to compute the induced current, which has been, in fact, followed by Jackiw and Nair in Ref. [13]. Starting with the effective action $S$ of Taylor and Wong, eq. (4.5), they have constructed $j_{a}^{\mu}$ as the first derivative of $S$, i.e. eq. (4.4), with $a_{a}^{0}\left[A_{+}\right] \equiv \delta W / \delta A_{+}^{a}$ determined from the gauge-invariance condition on $S$, eq. (4.1), as in the original work of Taylor and Wong. In order to recognize that the expression for $j_{a}^{\mu}$ obtained in [13] is the same as ours, we follow Ref. [7] and replace eq. (4.1) with an equivalent equation for the new function $a_{-} \equiv 2 a_{0}-A_{+}$:

$$
\partial_{+} a_{-}-\partial_{-} A_{+}+i g\left[A_{+}, a_{-}\right]=0 .
$$

Then, the current (4.4) becomes

$$
j^{\mu}(x)=3 \omega_{p}^{2} \int \frac{d \Omega}{4 \pi} v^{\mu}\left(a_{0}-A_{0}\right)=\frac{3}{2} \omega_{p}^{2} \int \frac{d \Omega}{4 \pi} v^{\mu}\left(a_{-}-A_{-}\right) .
$$

We recognize in eqs. (4.10) and (4.11) the expression of $j^{\mu}$ proposed in Ref. [13].

On the other hand, it can be easily verified using eqs. (2.7) and (3.13) that $a_{-}$just defined is also equal to $v^{\prime} \cdot a$. This is not surprising, since eq. (4.10) also follows from eq. (3.14) for $a^{\mu}$ after multiplication with $v_{\mu}^{\prime}$. Let us further define $a_{+} \equiv v \cdot a$. Because of eqs. (2.7) and (3.13), we have $a_{+}=A_{+}$(i.e., $v \cdot a=v \cdot A$, as already noticed after eq. (3.19)), so that eq. (4.10) may be finally written as

$$
\partial_{+} a_{-}-\partial_{-} a_{+}+i g\left[a_{+}, a_{-}\right]=0
$$

which coincides with the projection of the zero-curvature condition (3.17) onto the hyperplane defined by the 4-vectors $v^{\mu}$ and $v^{\prime \mu}$; that is, eq. (4.12) is the same as $v_{\mu} f^{\mu \nu} v_{\nu}^{\prime}=0$. If we recall that $a_{+}=a_{0}-\mathbf{v} \cdot \mathbf{a}$ and $a_{-}=a_{0}+\mathbf{v} \cdot \mathbf{a}$, we see that the gauge structure defined by eq. (4.12) involves the temporal $\left(a_{0}\right)$ and the longitudinal $(\mathbf{v} \cdot \mathbf{a})$ components of $a_{\mu}$, but not its two transverse components. This equation represents the starting point of the relation to the Chern-Simons theory explored in Refs. [7].

The main difference between the two derivations of $j_{\mu}$ outlined above concerns the method used for solving eq. (4.10) (or, equivalently, eq. (2.6) for $W^{0}$ ). We get the solution directly in Minkovski space, where retarded conditions are easily implemented (see eq. (2.9) above). The authors of Ref. [13] solve eq. (4.10) in Euclidian space-time, using technics borrowed from the study of the Chern-Simons theory. They obtain thus $a_{-}$ as a power series in $A_{+}$[7]. In order to get the retarded current, an analytic continuation has to be performed, to ensure the correct $i \epsilon$ prescription [13].

The functions $W^{\mu}$ have been found useful in the construction of the energy-momentum tensor for the gauge fields [14, 17], and also in the construction of a manifestly gauge invariant expression for the effective action. Such an expression was proposed first by Braaten 
and Pisarski[5]. Their expression reads (with $\left.(\tilde{D} F)^{a} \equiv[D, F]^{a}\right)$

$$
S=\frac{3}{2} \omega_{p}^{2} \int \frac{d \Omega}{4 \pi} \int d^{4} x \int d^{4} y \operatorname{Tr}\left[F_{\mu \lambda}(x)\left\langle x\left|\frac{v^{\mu} v_{\nu}}{(v \cdot \tilde{D})^{2}}\right| y\right\rangle F^{\nu \lambda}(y)\right],
$$

and has been rederived since by Frenkel and Taylor [11] (who inferred $S$ from a study of the forward scattering amplitudes for quarks and gluons on "soft" gauge fields), and by us (in Ref. [10], we have shown explicitly that the functional derivative of (4.13) gives the correct induced current). The functional (4.13) is well defined only on $\mathcal{R}$, where it coincides with [14]

$$
S=-\frac{3}{2} \omega_{p}^{2} \int d^{4} x \int \frac{d \Omega}{4 \pi} \operatorname{Tr} W^{\mu}(x ; v) W_{\mu}(x ; v) .
$$

By using eqs. (3.13) and (3.18), we can write

$$
W^{\mu}(x ; v)=\frac{i}{g}\left(D^{\mu} V\right) V^{-1}
$$

Thus, an alternative expression for $S$ is

$$
S=-\frac{3}{2} \frac{\omega_{p}^{2}}{g^{2}} \int d^{4} x \int \frac{d \Omega}{4 \pi} \operatorname{Tr}\left(D_{\mu} V\right)\left(D^{\mu} V\right)^{\dagger}
$$

which also appears in Ref. [15.

Let us finally consider the energy $\mathcal{E}$ of a gauge field configuration in the plasma. Different approaches has been proposed recently to compute this quantity[14, 15, 16, 17], and the various results obtained are not related in an obvious way. In Ref. [17, we have calculated $\mathcal{E}$ by integrating the field equations of motion (2.1). One of our results may be written in the form $\mathcal{E}=\mathcal{E}_{Y M}+\mathcal{E}_{\text {ind }}$, where $\mathcal{E}_{Y M}$ is the standard Yang-Mills contribution,

$$
\mathcal{E}_{Y M} \equiv \int d^{3} x \frac{1}{2}\left(\mathbf{E}^{a}(x) \cdot \mathbf{E}^{a}(x)+\mathbf{B}^{a}(x) \cdot \mathbf{B}^{a}(x)\right),
$$

$\left(B_{a}^{i}(x) \equiv-(1 / 2) \epsilon^{i j k} F_{a}^{j k}(x)\right)$, while

$$
\mathcal{E}_{\text {ind }} \equiv 3 \omega_{p}^{2} \int d^{3} x \int \frac{d \Omega}{4 \pi} \operatorname{Tr} W^{0}(x ; v) W^{0}(x ; v),
$$

may be interpreted as the polarization energy of the plasma, that is, the energy transferred by the gauge fields to the plasma constituents 17. According to these expressions, the field energy is obviously positive. A similar conclusion has been reached by Nair 16, who derived a Hamiltonian for the soft gauge fields by exploiting the analogy with the ChernSimons theory. The resulting energy functional is positive when evaluated for gauge fields which satisfy Gauss's law. Nair's expression for $\mathcal{E}$ reads $\mathcal{E}=\mathcal{E}_{Y M}+\mathcal{E}_{N}$, with

$$
\mathcal{E}_{N} \equiv \frac{3}{4} \frac{\omega_{p}^{2}}{g^{2}} \int d^{3} x \int \frac{d \Omega}{4 \pi} \operatorname{Tr}\left\{\left[D_{0}, G\right]\left[D_{0}, G^{\dagger}\right]+[\mathbf{v} \cdot \mathbf{D}, G]\left[\mathbf{v} \cdot \mathbf{D}, G^{\dagger}\right]\right\}
$$


and involves the auxiliary matrix field $G(x, \mathbf{v}) \in S U(N)$, with the property $G(x, \mathbf{v})=$ $G^{\dagger}(x,-\mathbf{v})$.

We show now that, in spite of formal differences, the energy densities in eqs. (4.18) and (4.19) are, in fact, the same. To do that, we first replace $D_{0}=\left(D_{-}+D_{+}\right) / 2$ and $\mathbf{v} \cdot \mathbf{D}=\left(D_{-}-D_{+}\right) / 2\left(\right.$ where $D_{+} \equiv v \cdot D$ and $\left.D_{-} \equiv v^{\prime} \cdot D\right)$. We thus get, instead of (4.19),

$$
\mathcal{E}_{N} \equiv \frac{3}{8} \frac{\omega_{p}^{2}}{g^{2}} \int d^{3} x \int \frac{d \Omega}{4 \pi} \operatorname{Tr}\left\{\left[D_{-}, G\right]\left[D_{-}, G^{\dagger}\right]+\left[D_{+}, G\right]\left[D_{+}, G^{\dagger}\right]\right\}
$$

By changing $\mathbf{v} \rightarrow \mathbf{- v}$ in the second term inside the curly braces, we see that it gives the same contribution as the first one after angular integration. Furthermore, we note that the equations of motion for $G(x, \mathbf{v})$, as written down in Ref. [16], are implicitly solved by

$$
a_{-}=G^{-1} A_{-} G-\frac{i}{g} G^{-1} \partial_{-} G
$$

which relates $G$ to our function $a_{-} \equiv v^{\prime} \cdot a$. Writing this equation in the form

$$
a_{-}-A_{-}=-\frac{i}{g} G^{-1}\left[D_{-}, G\right]=\frac{i}{g}\left[D_{-}, G^{\dagger}\right] G
$$

we conclude that eq. (4.20) is equivalent to

$$
\mathcal{E}_{N} \equiv \frac{3}{4} \omega_{p}^{2} \int d^{3} x \int \frac{d \Omega}{4 \pi} \operatorname{Tr}\left\{\left(a_{-}-A_{-}\right)\left(a_{-}-A_{-}\right)\right\}
$$

where any direct reference to the field $G$ has disappeared. It is then sufficient to recall that $a_{-}-A_{-}=2 W^{0}$ to see that $\mathcal{E}_{N}$, as given by eq. (4.23), is identical to our expression (4.18) for $\mathcal{E}_{\text {ind }}$. Remark that in the arguments above we did not need the explicit expression of $G$. However, if one recalls that $a_{-}=(i / g)\left(\partial_{-} V\right) V^{-1}$ (see eq. (3.18)), with $V(x ; v)$ defined by (2.13), one can easily verify that eq. (4.21) is solved by $G(x, \mathbf{v})=V\left(x ; v^{\prime}\right) V^{\dagger}(x ; v)$.

\section{Conclusions}

The classical features of the HTL's reflect the long-wavelength, collective behaviour of the plasma particles, namely, their average motion over distances which are large compared to their mean separation. The coherent motion of the particles is described, to leading order in $g$, by density matrices obeying simple kinetic equations. In the abelian case, the kinetic equation is the linearized Vlasov equation and the elementary dynamics is essentially that of the Lorentz equation which governs the motion of a classical charged particle in an electromagnetic field. In the non-abelian case, the distribution functions are matrices 
in color space and their time dependence involves in addition a "precession" in color space. We have shown that a remarkably simple gauge structure emerges from our kinetic equations once one assumes that the plasma particles are not significantly deviated by the background field. Remarkably, this gauge structure determines completely the collective dynamics of the particles at the order of the HTL's approximation. This explains why the requirement of gauge symmetry was, in fact, a sufficient condition to obtain the effective action generating the HTL's [4], as well as the related field equations [7, 13].

\section{Acknowledgements}

We would like to thank V.P. Nair for useful correspondance concerning his work on the energy of gauge field configurations 16.

\section{References}

[1] R.D. Pisarski, Phys. Rev. Lett.63 (1989) 1129.

[2] E. Braaten and R.D. Pisarski, Nucl. Phys. B337 (1990) 569; ibid. B339 (1990) 310; Phys. Rev. Lett.64 (1990) 1338.

[3] J. Frenkel and J.C. Taylor, Nucl. Phys. B334 (1990) 199.

[4] J.C.Taylor and S.M.H.Wong, Nucl. Phys. B346 (1990) 115.

[5] E. Braaten and R. Pisarski, Phys. Rev. D45 (1992) 1827.

[6] J. Frenkel and J.C. Taylor, Nucl. Phys. B374 (1992) 156.

[7] R. Efraty and V.P. Nair, Phys. Rev. Lett.68 (1992) 2891; Phys. Rev. D47 (1993) 5601.

[8] J.P. Blaizot and E. Iancu, Nucl. Phys. B390 (1993) 589.

[9] J.P. Blaizot and E. Iancu, Phys. Rev. Lett.70 (1993) 3376.

[10] J.P. Blaizot and E. Iancu, Saclay preprint T93/064, to appear in Nucl. Phys. B.

[11] U. Heinz, Phys. Rev. Lett.51 (1983) 351; see also H.-Th. Elze and U. Heinz, Phys. Repts.183 (1989) 81. 
[12] L.D. Landau and E.M. Lifshitz, The Classical Theory of Fields, (Pergamon Press, Oxford, 1975).

[13] R. Jackiw and V.P. Nair, Phys. Rev. D48 (1993) 4991.

[14] H.A. Weldon, Canadian J. Phys. 71 (1993) 300.

[15] F.T. Brandt, J. Frenkel and J.C. Taylor, Nucl. Phys. B410 (1993) 3.

[16] V.P. Nair, Phys. Rev. D48 (1993) 3432.

[17] J.P. Blaizot and E. Iancu, "Energy-Momentum Tensors for the Quark-Gluon Plasma", Saclay preprint T94/03, January 94, to appear in Nucl. Phys. B.

[18] R. Jackiw, Q. Liu and C. Lucchesi, MIT preprint CTP-2261, November 1993. 